\title{
Hippocampal CA3 Transcriptional Modules Associated with Granule Cell Alterations and Cognitive Impairment in Refractory Mesial Temporal Lobe Epilepsy Patients
}

\section{Silvia Bando}

Faculdade de Medicina da Universidade de São Paulo

\section{Fernanda Bertonha}

Faculdade de Medicina da Universidade de São Paulo

Luciana Pimentel-Silva

State University of Campinas

João Mansano de Oliveira

Faculdade de Medicina da Universidade de São Paulo

\section{Marco Duarte Carneiro}

Faculdade de Medicina da Universidade de São Paulo

\section{Mariana Manoel Oku}

Faculdade de Medicina da Universidade de São Paulo

\section{Hung-Tzu Wen}

Hospital das Clínicas da Faculdade de Medicina da Universidade de São Paulo

\section{Luiz Martins Castro}

Faculdade de Medicina da Universidade de São Paulo

Carlos Moreira-Filho ( $\nabla$ carlos.moreira@hc.fm.usp.br)

Faculdade de Medicina da Universidade de São Paulo

\section{Research Article}

Keywords: epilepsy, patients, drug, RMTLE

Posted Date: January 13th, 2021

DOI: https://doi.org/10.21203/rs.3.rs-142725/v1

License: (c) (1) This work is licensed under a Creative Commons Attribution 4.0 International License. Read Full License 


\section{Abstract}

In about a third of the patients with epilepsy the seizures are not drug-controlled. The current limitation of the antiepileptic drug therapy derives from an insufficient understanding of epilepsy pathophysiology. In order to overcome this situation, it is necessary to consider epilepsy as a disturbed network of interactions, instead of just looking for changes in single molecular components. Here, we studied CA3 transcriptional signatures and dentate gyrus histopathologic alterations in hippocampal explants surgically obtained from 57 RMTLE patients submitted to corticoamygdalohippocampectomy. By adopting a systems biology approach, integrating clinical, histopathological, and transcriptomic data (weighted gene co-expression network analysis), we were able to identify transcriptional modules highly correlated with age of disease onset, cognitive dysfunctions, and granule cell alterations. The enrichment analysis of transcriptional modules and the functional characterization of the highly connected genes in each trait-correlated module allowed us to unveil the modules' main biological functions, paving the way for further investigations on their roles in RMTLE pathophysiology. Moreover, we found 15 genes with high gene significance values which have the potential to become novel biomarkers and/or therapeutic targets in RMTLE.

\section{Introduction}

Epilepsy affects 50 million people worldwide and is characterized by unprovoked recurrent seizures due to abnormal neuronal discharge, and by the neurobiological, cognitive, and psychological consequences of seizure recurrence ${ }^{1}$. Mesial temporal lobe epilepsy (MTLE), the commonest focal epilepsy in adults, involves the medial structures of the temporal lobe and hippocampal sclerosis (HS) constitutes its most frequent pathological abnormality ${ }^{2}$. MTLE is associated with a history of prolonged febrile seizures in early childhood or other initial precipitating injuries (IPI) ${ }^{3}$. About $40 \%$ of the MTLE patients with a history of febrile seizures develop refractory (drug-resistant) epilepsy (RMTLE) ${ }^{4}$. Furthermore, early IPI and early epilepsy onset (i.e., in the first years of life) are associated respectively with severe hippocampal neuronal loss ${ }^{5}$ and refractory epilepsy ${ }^{6}$. Refractory epilepsy increases the risk of psychosocial dysfunction, cognitive decline, and sudden unexpected death of patients ${ }^{7}$.

RMTLE patients can benefit from surgical treatment ${ }^{8}$, and hippocampal explants obtained at epilepsy surgery are a valuable material for investigating the cellular, molecular and genomic mechanisms underlying refractory epilepsy. Studies in surgical specimens helped to unveil the relevance of hippocampal areas with regard to the temporal lobe epilepsies (TLE) progression and, particularly, of the dentate gyrus (DG) in biological processes related to the startup and to the end stages of the disease ${ }^{9}$. Pathomorphological studies showed compromised neurogenesis and significant DG cell loss in RMTLE patients ${ }^{10,11}$ and in vitro and animal model assays showed that the improper circuit control of DG-CA3 synapses leads to epilepsy, since DG acts as a "gate", protecting hippocampal circuits from overexcitation ${ }^{12,13}$. Multi-omics analyses performed in the pilocarpine rat model of MTLE suggested enhanced epileptogenesis in the CA3 region when compared to the DG, with most of the transcriptional 
and protein expression alterations occurring in $\mathrm{CA}^{14}$. Additionally, the integrative study of $\mathrm{CA} 3$ transcriptional signatures and DG histopathology in hippocampal explants surgically obtained from RMTLE patients allowed our group, and others, to portrait the disease as a disturbed network of genegene interactions ${ }^{15-19}$.

On the other hand, even considering the good results attained by the surgical treatment for refractory epilepsy, only a minority of patients with RMTLE are ever referred to epilepsy surgery, and often too late to prevent serious disabilities ${ }^{20}$. This situation reinforces the demand for identifying genomic mechanisms that can be targeted for novel preventive and drug-based therapeutic interventions ${ }^{21}$. The transcriptome analysis pipeline for this task usually involves building gene co-expression networks, finding and functionally characterizing the transcriptional modules correlated to particular traits, their hub genes, which are associated to specific cellular processes or link different biological processes, and their genes significantly correlated with a particular trait ${ }^{22,23}$. Here, by using this pipeline and integrating demographic, clinical, cognitive, histopathological and genomic data, we investigated: i) if in patients with RMTLE the cognitive function impairments and DG histological alterations could be correlated with CA3 transcriptional modules; ii) if the genes with high connectivity (hubs) or high gene significance in the trait-correlated modules were related to epilepsy-relevant cellular and molecular mechanisms.

\section{Results}

We studied 57 RMTLE patients with unilateral mesial temporal sclerosis (MTS) who had been submitted to corticoamygdalohippocampectomy (Supplementary Table S1 online). Patients' cognitive function was assessed before surgery. Histological analysis was performed in resected hippocampi specimens to diagnose and determine the pattern of hippocampal sclerosis (HS) and to assess the grades of cell loss, dispersion and bilamination. During epilepsy surgery tissue explants from CA3 were obtained for genomic studies. Weighted Gene Co-expression Network Analysis (WGCNA) ${ }^{22}$ was used for constructing the module-trait relationships considering demographic, clinical, cognitive, histopathologic and genomic data (Fig. 1).

\section{Cognitive function evaluation}

Forty-four RMTLE patients and two control groups totaling 60 volunteers - one control subgroup for memory and executive function evaluation $(n=40)$, and another for language evaluation $(n=20)$ - were matched for age, gender, education, and IQ scores (Supplementary Table S2 online). Patients and controls underwent a cognitive test battery for language, memory, and executive function (Supplementary Table S3 online). The patient's group was divided into two subgroups: i) LHS subgroup ( $n=27)$, encompassing left HS patients ii) RHS subgroup $(n=17)$ for right HS patients. Cognitive tests data from patients and controls were submitted to ROC analysis for identifying which tests were classificatory and obtaining the corresponding cut-off values. Thereafter, a comparative analysis of tests' hits was accomplished (Supplementary Table S3 online). The LHS subgroup achieved less tests hits $(p<0.05)$ in 
semantic and phonological fluency, verbal/proper noun naming, RAVLT total and late recall, logical memory, ROCF, Stroop test, and FAS test (Supplementary Table S3 online). The RHS subgroup achieved less tests hits $(p<0.05)$ in semantic fluency, object/verbal/proper noun/responsive naming, RAVLT immediate recall and late recognition, RVDLT and facial recognition, ROCF, Stroop test, and FAS test (Supplementary Table S3 online). These results were used for scoring failures in memory, language, and executive function impairment (Supplementary Table S4 online). The patients were then classified in two subgroups regarding cognitive impairment: severe, where total score > average score, or mild, where total score $\leq$ average score (Supplementary Tables S1 and S4 online). Fisher's exact test for severe cognitive impairment showed no significant probability $(p>0.05)$ for laterality.

\section{Histopathology}

Forty-three resected hippocampi were used for histopathological analysis (Supplementary Table S1 online). The HS types were classified according to Blümcke et al. ${ }^{24}$. Semiquantitative assessment was performed on dentate gyrus abnormalities, particularly cytoarchitectural disorganization: granule cell loss (GCL), dispersion (GCD), and bilamination (GCB). Table 1 shows the frequency distribution of histological features. HS ILAE type 1 (severe CA1 and CA4 neuronal loss and gliosis) was the most frequent type (90.9\%), followed by HS ILAE types 2 (CA1 neuronal loss and gliosis), and one no-HS (preserved cell density and gliosis only). GCL was observed in 42 samples, being GCL grade 2 the most frequent type (41.9\%) followed by grades 3 and $0-1$. GCD was observed in 38 samples and the most frequent type was GCD grade $3(37.2 \%)$, followed by grades 1,2 and 0 . GCB was present in 15 out of 43 samples (34.9\%). 
Table 1

Demographic and clinical characteristics of 43 RTMLE patients and histological features of the patients' hippocampal samples.

\begin{tabular}{|c|c|c|c|}
\hline \multicolumn{2}{|l|}{ Phenotypic characteristics } & \multirow{2}{*}{$\begin{array}{l}\text { Number } \\
21\end{array}$} & \multirow{2}{*}{$\begin{array}{l}\% \\
48.8\end{array}$} \\
\hline Gender & Male & & \\
\hline & Female & 22 & 51.2 \\
\hline \multirow[t]{2}{*}{ Severe memory impairment } & Left HS $(n=18)$ & 7 & 38.9 \\
\hline & Right HS ( $n=12)$ & 6 & 50.0 \\
\hline \multirow[t]{2}{*}{ Severe language impairment } & Left HS $(n=18)$ & 5 & 27.8 \\
\hline & Right HS ( $n=12)$ & 5 & 41.7 \\
\hline \multirow[t]{2}{*}{ Severe executive function impairment } & Left HS $(n=17)$ & 7 & 35.3 \\
\hline & Right HS ( $n=12)$ & 6 & 50.0 \\
\hline \multirow[t]{2}{*}{ Brain side affected by HS } & Left & 25 & 58.1 \\
\hline & Right & 18 & 41.9 \\
\hline \multirow[t]{2}{*}{ IPI status } & Febrile & 15 & 34.9 \\
\hline & Afebrile & 27 & 62.8 \\
\hline \multirow[t]{2}{*}{ Age at IPI } & Early ( $\leq 5$ yrs) & 25 & 58.1 \\
\hline & $>5$ yrs & 18 & 41.9 \\
\hline \multirow[t]{2}{*}{ Age at disease onset } & Early ( $\leq 5$ yrs) & 8 & 18.6 \\
\hline & $>5$ yrs & 35 & 81.4 \\
\hline \multirow[t]{3}{*}{ HS ILAE type } & 1 & 40 & 90.9 \\
\hline & 2 & 3 & 6.8 \\
\hline & No-HS & 1 & 2.3 \\
\hline \multirow[t]{3}{*}{ Grade of GCL } & 0 to 1 & 11 & 25.6 \\
\hline & 2 & 18 & 41.9 \\
\hline & 3 & 14 & 32.6 \\
\hline \multirow[t]{4}{*}{ Grade of GCD } & 0 & 5 & 11.7 \\
\hline & 1 & 13 & 30.2 \\
\hline & 2 & 9 & 20.9 \\
\hline & 3 & 16 & 37.2 \\
\hline
\end{tabular}




\begin{tabular}{|lllll|}
\hline Phenotypic characteristics & & Number & $\%$ \\
\hline Presence of GCB in the dentate gyrus & Yes & 15 & 34.9 \\
\hline & No & 28 & 65.1 \\
\hline
\end{tabular}

\section{Clinicopathological correlation analyses}

Correlation analyses for the LHS and RHS subgroups were performed between pairs of selected demographical, clinical and pathological variables: gender, age at surgery, age at IPI, age of epilepsy onset, cognition (memory, language, and executive function) and histological features (HS type, grade of GCL or GCD, and presence of GCB). A statistically significant and positive correlation was found for GCL and memory impairment grades $(r=0.47, p=0.02)$ in the LHS subgroup.

\section{Weighted Gene Co-expression Network Analysis}

Total RNA samples were obtained from the hippocampal CA3 region of 22 RMTLE patients (Supplementary Table S1 online) and used for DNA microarray hybridizations. The normalized gene expression data of 8,104 GO (Gene Ontology) annotated genes were used for network construction and module identification by WGCNA. Pearson's correlation coefficient was used for obtaining gene coexpression network and dynamic tree cut algorithm was used for dendrogram`s branch selection for module identification. Eleven transcriptional modules were identified. The module sizes ranged from 215 (green yellow module) to 1,795 (turquoise module) genes (Fig. 2a-b). The resulting eigengene network presented two meta-modules, here named I and II, as displayed in Fig. 2c.

\section{Module-trait correlation analyses}

We performed two module-trait correlation analyses: one for the demographic, clinical, and histopathological traits including all patients in LHS and RHS subgroups and another for cognitive function, conducted separately for LHS and RHS subgroups (due to the significant correlation between GCL and severe memory impairment in LHS), where a statistically significant module-trait correlation was found only for LHS patients. All transcriptional modules presented significant correlation $(p<0.05)$ with at least one trait and nine of these modules - four in the meta-module I and five in the meta-module II showed high correlation values $(r>|0.50|)$ with at least one trait, as next described (Fig. 2c;

Supplementary Figs. S1 and S2 online). In the meta-module I the blue module is positively correlated with early IPI (E-IPI), granule cell alterations (GCL grade 3), and severe executive function impairment (SEFI), whereas in the meta-module II the turquoise module is negatively correlated with those three traits. The yellow module in the meta-module I is positively correlated with GCD2. Inversely, the purple module in meta-module II is negatively correlated with that trait. The red module - in a separate branch in the metamodule II (Fig. 2C) - is positively correlated with early disease onset (E-onset). Interestingly, the metamodule I harbors modules positively correlated with severe phenotypes (GCL3, GCD2, E-IPI, SEFI) and negatively correlated with mild phenotypes (GCL0-1, GCD1), whereas in meta-module II these correlations are quite inverted. 


\section{Functional characterization of modules}

Transcriptional modules often represent biological processes can be phenotype specific ${ }^{25}$. The functional enrichment among the genes within a module is widely used for disclosing its biological meaning ${ }^{25}$. Here we used a web-based GO enrichment analysis tool ${ }^{26}$ and found that between 18 and $57 \%$ of the genes within the modules highly correlated with at least one trait were significantly $(p<0.05)$ over-represented in GO biological processes (BP) terms (Fig. 3). These terms were arbitrarily grouped in two major functional categories: i) molecular and cellular processes relevant to MTLE and brain functioning ii) other related molecular and cellular processes (Fig. 3, Supplementary Table S5 online). The complete list of the BP terms found for all modules is presented in Supplementary Datasheet S1 online. Three transcriptional modules - red, turquoise and blue - have 30 percent or more of their genes over-represented in the category "molecular and cellular processes relevant to MTLE and brain functioning" (Fig. 3;

Supplementary Table S5 online). Noteworthy, the blue and turquoise modules are associated with more traits than any of the other modules (Fig. 2C), and the red module - highly and positively correlated with Eonset - has 163 genes associated to processes linked to MTLE, of which 105 (Supplementary Table S5 online) are related with neuron/excitability processes (neuron, glia, axon, myelination, excitability, and synapse).

\section{Categorization of high-hierarchy $(\mathrm{HH})$ genes}

Highly connected genes hold the whole transcriptional network together and are either associated to specific cellular processes or link different biological processes ${ }^{23}$. Thus, we used connectivity measures for the hierarchical categorization of genes (see Methods) in the modules highly correlated with at least one trait ${ }^{22}$. $\mathrm{HH}$ genes were classified in one of three hierarchical categories according to the number of gene-gene links: i) intramodular hub (iHub), a gene highly connected with other genes in the same module; ii) high hub (Hhub), a hub that also has a high whole network connectivity; and iii) intermodular hubs (eHub), a gene with low number of intramodular links but high whole network connectivity. Additionally, the gene significance (GS) value and the p-value obtained for each $\mathrm{HH}$ gene-trait correlation were used for obtaining the gene expression profiles positively (hyper-expression) or negatively (hypoexpression) correlated with histological and/or cognitive traits. A total of $76 \mathrm{HH}$ genes were thereby found and categorized (Supplementary Figs. S3 and S4 online), being distributed into nine modules, as depicted in Figs. 4 and 5, corresponding to meta-modules I and II, respectively. 
Table 2

HGS genes identified in modules associated with at least one trait: GCL, GCD, GCB, with severe memory impairment, or early/late disease onset. ${ }^{\mathrm{L}}$ indicates LHS group; *genes that presented highest GS and MM values; in bold genes that are also iHubs.

\begin{tabular}{|lll|}
\hline Trait & Gene & Go database: biological process; molecular function \\
\hline GCL & & \\
\hline Blue & AMFR & protein ubiquitination; learning or memory \\
\hline Black & CHSY1* & chondroitin sulfate biosynthetic process \\
\hline Turquoise & ISYNA1 & inositol biosynthetic process \\
\hline Brown & LGR4 & positive regulation of canonical Wnt signaling pathway \\
\hline Black & MGAT2* & protein N-linked glycosylation via asparagine \\
\hline Brown & PLTP & phospholipid transport \\
\hline GCD & & \\
\hline Blue & ERBB4 & protein tyrosine kinase activity \\
\hline Brown & SMARCAD1 & chromatin remodeling \\
\hline Blue & DHX9 & DNA helicase activity \\
\hline Turquoise & MDH2 & RNA binding \\
\hline GCB & & \\
\hline Blue & HYOU1 & cellular response to hypoxia \\
\hline Severe memory impairment & \\
\hline Brown & ASNSD1 & glutamine metabolic process; asparagine biosynthetic process \\
\hline Disease onset & FLDLR & reelin-mediated signaling pathway \\
\hline Red & fibroblast growth factor-activated receptor activity \\
\hline & epidermal growth factor receptor signaling pathway \\
\hline
\end{tabular}

\section{Functional interpretation of $\mathrm{HH}$ genes in trait-associated modules}

The $\mathrm{HH}$ genes were interpreted in the context of the two eigengene network meta-modules. The metamodules - identifiable as branches in the eigengene dendrogram - are sets of modules and genes with stronger relationship and reveal a higher order organization among gene co-expression modules ${ }^{27}$. Metamodules are biologically significant ${ }^{22}$ and the functional enrichment analysis of their modules may help 
to reveal their meaning (Fig. 3). In Figs. 4 and 5, related to meta-modules I and II, respectively, the expression value profile of the $\mathrm{HH}$ genes corresponds, in a large measure, to the module-trait correlations above mentioned (Fig. 2c).

\section{$\mathrm{HH}$ genes in the meta-module I}

In the blue module all HH genes are positively correlated with E-IPI, GCL3 and SEFI (Fig. 4) and two of its Hhubs, ITGB8 and UQCRB, are involved in brain angiogenesis ${ }^{28}$ and in angiogenesis induction ${ }^{29}$, respectively. This is rather expectable since dysregulated angiogenesis and vascular remodeling are hallmarks of the epileptic brain ${ }^{30}$. Two other blue module genes, the Hhub KIF1B and the eHub YAP1, were shown to be essential for survival of hippocampal neurons and their hyper-expression in the sclerotic hippocampi may well constitute a compensatory mechanism ${ }^{31,32}$.

In the yellow module nine out of the eleven $\mathrm{HH}$ genes are positively correlated with GCD2 (Fig. 4). The HHub TANC2 and the eHub SLC25A18, both hyper-expressed, belong to the glutamatergic excitatory system ${ }^{33,34}$, whose dysfunction leads to hyperexcitatory neural networks and neurotoxicity ${ }^{35}$. The iHub SPOCK1, also hyper-expressed, encodes the proteoglycan testican, is expressed in hippocampal pyramidal neurons and has a functional role in reactive gliosis ${ }^{36}$. Reactive gliosis is a quite common finding in drug resistant TLE ${ }^{24}$. Noteworthy, the Hhub CYB5D2, that codes for neuferricin and is involved in hippocampal neurogenesis ${ }^{37}$, is negatively correlated with GCD2.

In the green yellow module the only gene positively correlated with GCD1 is the eHub FBXW7, which exerts a protective role against glutamate receptor-mediated excitotoxicity ${ }^{38}$. Finally, in the magenta module all $\mathrm{HH}$ genes but one are negatively correlated with GCL0-1, the exception being the eHub RAB32 whose upregulation is a marker for neuroinflammatory lesions ${ }^{39}$.

\section{HH genes in the meta-module II}

In this meta-module five out of seven modules harbor $\mathrm{HH}$ genes. The turquoise module is the largest in number of genes (Fig. 2b) and its module-trait correlation is almost the inverse of that found for the blue module in meta-module I (Fig. 2c). Most of the HH genes in this module are positively correlated with the mild phenotype GCL0-1 but negatively correlated with the severe phenotypes E-IPI, GCL3, and SEFI (Fig. 5). Two genes are indeed exemplary in this context: PIK3C3, essential for CNS neuronal homeostasis ${ }^{40}$ and for axon growth in hippocampal neurons ${ }^{41}$, and $D C P 1 B$, a potential regulator of human memory performance through its interaction with miR-138-5 $\mathrm{p}^{42}$, are both negatively correlated with GCL3 and SEFI.

In the brown module all HH genes are negatively correlated with GCL3 and SMI (Fig. 5). This module harbors the eHubs LGR4 (also an HGS gene), a positive regulator of the canonical Wnt/ $\beta$ catenin signaling pathway ${ }^{43}$ and EIF4E, a mTOR downstream effector ${ }^{44}$. The Wnt/ $\beta$ pathway is a principal positive regulator of adult hippocampal neurogenesis and its disruption contributes to the functional and structural abnormalities of temporal lobe epilepsy ${ }^{45}$. Conversely, an increased mTOR activation was 
observed in the sclerotic hippocampi from patients with RMTLE ${ }^{46}$ and EIF4E was recently identified as therapeutic target in intractable epilepsy ${ }^{47}$. Here we found that LGR4 and EIFE4 were both negatively correlated with GCL3.

In the black and purple modules the HH genes are negatively correlated with GCL3 and GCD2, respectively. The black module harbors the iHub MGAT2 - also an HGS gene -that controls N-glycan branching and is required for normal neuronal development and viability ${ }^{48}$. This gene is markedly hypoexpressed in the GCL3 hippocampi (Fig. 6). In the purple module it is worth mentioning the HHub SDK2, which encodes a sidekick molecule that mediates neuronal cell-cell adhesion ${ }^{49}$.

The red module has six $\mathrm{HH}$ genes, all involved in relevant brain and neuronal functions. Four of these genes are positively correlated with E-onset (Fig. 5): the HHub OLFM1 codes for olfactomedin and promotes hippocampal axon growth after axonal injury ${ }^{50}$; the iHub ST8SIA3 is involved in mediating sialylation and neuronal plasticity ${ }^{51}$, and increased polysialylation and neuronal circuitry remodeling were observed in the hippocampus and entorhinal cortex of RMTLE patients ${ }^{52}$; the iHub, SVOP, codes for a synaptic vesicle transport protein structurally related to SV2A, the target of the anti-epileptic drug Levetiracetam ${ }^{53,54}$; the eHub $N C D N$ encodes neurochondrin (norbin), which promotes neurite outgrowth, regulates hippocampal neurogenesis, modulates the metabotropic glutamate receptor 5 , and was found to be downregulated in the hippocampus of intractable TLE patients ${ }^{55}$. Regarding the two negatively correlated genes: the eHub ERBB3 mediates hippocampal neurogenesis through neuregulin-1/ERBB3 signaling, an essential process for memory ${ }^{56}$; and the eHub SYNJ2, that encodes synaptojanin-2 and is involved in astrocyte morphology remodeling ${ }^{57}$, was shown to influence human cognitive abilities ${ }^{58}$.

\section{Functional interpretation of HGS (High Gene Significance) genes}

Genes presenting high GS and high Module Membership (MM) values are considered HGS genes (see Methods) in a module and are significantly correlated with a particular trait ${ }^{22}$. Gene-trait correlations and biological functions for the HGS genes appear in Table 2, and the corresponding statistical data is shown in Supplementary Table S6 online. The functional interpretation of HGS genes was based on GO and ad hoc annotations ${ }^{59}$. The gene expression plots for HGS genes, the relationships between gene expression values and traits, and the statistical significance of these relationships are displayed in Fig. 6.

Six out of the 15 HGS genes are correlated with GCL (Table 2) and their expression levels show significant differences between GCL grades (Fig. 6). Two of these genes, AMFR (alias GP78) and PTLP, are involved in learning and memory. AMFR encodes a receptor of the neurotrophic factor neuroleukin and its hippocampal expression is correlated with learning and memory in animal models ${ }^{60,61}$. PTLP codes for the plasma phospholipid transfer protein, which exerts a neuroprotective role through its ability to deliver vitamin E to the brain, and PTLP-KO mice have impaired memory and learning capabilities ${ }^{62}$. In GCL3 sclerotic hippocampi AMFR expression is increased from GCL3 to GCL0-1 and PTLP expression - 
neuroprotective - is decreased (Fig. 6). Two other genes, CHSY1 and ISYNA1 (alias INOS), are involved in the biosynthesis of chondroitin sulfate and inositol, respectively. Chondroitin sulfate is important for the maintenance of hippocampal volume in patients with $\mathrm{TLE}^{63}$, and persistent elevated inositol levels were found in the hippocampus of rats presenting repeated seizures after spontaneous seizure induction ${ }^{64}$, and associated with pharmacoresistance in human TLE ${ }^{65}$. ISYNA1 expression is decreased from GCL3 to GCLO-1, and CHSY1expression is increased. Finally, LGR4 and MGAT2, which are also HH genes (Fig. 5), are relevant for brain and neuronal functioning. $L G R 4$ is a positive regulator of the canonical Wnt $/ \beta$ catenin signaling, which is disrupted both in the acute and chronic phases of TLE ${ }^{45,66}$. MGAT2 is required for the normal assembly of complex Asn-linked glycans, being essential for normal neurological development ${ }^{48}$. The expression of these two genes is decreased in the sclerotic hippocampi with GCL3.

Four HGS genes are correlated with GCD (Fig. 6). Two of these genes - ERBB4 and MHD2 - are directly involved in epilepsy and have increased expression in GCD2/GCD3. MHD2 codes for an RNA binding protein involved in the post-transcriptional downregulation of the sodium channel SNC1A expression in the hippocampus under seizure condition ${ }^{67}$. ERBB4 codes for an important receptor of Neuregulin 1 (NRG1) and is involved in neurodevelopment and synaptic plasticity ${ }^{68}$. Both NGR1 and ERBB4 have been implicated in animal model and human epilepsy and recent experimental data showed that these two proteins are overexpressed in symptomatic epilepsy, suggesting that the NRG1-ERBB4 signaling probably act as a homeostasis modulator protecting brain from aggravated epileptiform activity ${ }^{69}$. The other two genes correlated with GCD are SMARCAD1 and $D H X 9$, whose average gene expression levels vary across GCD grades (Fig. 6; Supplementary Table $S 6$ online). SMARCAD1 is a chromatin remodeling gene acting in mammal adult neurogenesis ${ }^{70}$ and $D H X 9$ codes for a DEAH-containing helicase which regulates DNA replication, transcription, translation, microRNA biogenesis, RNA processing and transport, and maintenance of genomic stability ${ }^{71}$.

HYOU1 is the only HGS gene correlated with GCB. Its overexpression prevents endoplasmic reticulum (ER) stress and rescues neurodegeneration ${ }^{72}$. ER stress has been recognized as a relevant etiological factor contributing to epilepsy-induced neuronal damage ${ }^{73}$. The expression of this gene is decreased in the sclerotic hippocampi with GCB.

The HGS gene ASNSD1 is involved in asparagine biosynthesis and its expression is decreased in our patients with severe memory impairment. Asparagine synthesis is essential for brain development and asparagine deficiency has been associated with deficits in learning and memory ${ }^{74}$.

Three HGS genes are correlated with disease onset and have diminished expression in our early-disease onset patients. Interestingly, these genes belong to the red module (Table 2) and are involved in synaptic plasticity and memory processes. VLDR codes for a component of the Reelin pathway regulating neuronal migration and synaptic plasticity in the hippocampus ${ }^{75}$. FGFR2 codes for the fibroblast growth factor receptor 2 and plays a role in the hippocampal short-term learning and long-term reference 
memory ${ }^{76}$, and $H B E G F$ codes for the heparin-binding EGF-like growth factor and plays a significant role in hippocampal synaptic plasticity and memory formation ${ }^{77}$.

\section{Discussion}

Module-trait correlation analyses revealed two meta-modules presenting high positive/negative correlations with severe (GCL3, GCD2, E-IPI, SMI, and SEFI) or mild (GCL0-1 and GCD1) phenotypes. In the meta-module I the transcriptional modules are positively correlated with the severe phenotypes and negatively with the mild phenotypes. Inversely, in the meta-module II all modules are negatively correlated with the severe phenotypes and positively with the mild phenotypes (Fig. 2c), except the red module, the only module that is positively correlated with E-onset and severe memory impairment. These correlations clearly indicate two distinct CA3 transcriptome profiles: one for severe and another for mild phenotypes. In addition, the functional enrichment analysis showed that the red and blue modules had many genes over-represented in neuron/excitability processes, such as neuron, glia, axon, myelination, excitability, and synapse (Fig. 3). In order to get a better understanding of how these modules are associated with DG alterations, cognitive impairment, and disease onset, we focused on the hierarchical characterization and functional interpretation of the genes within each module.

The blue and yellow modules (Fig. 4) harbor HH genes positively associated with pathophysiological mechanisms in epilepsy - such as vascular remodeling (ITGB8, UCQRB), the glutamatergic excitatory system (TANC2, SCLC25A18) and reactive gliosis (SPOCK1) - and with putative compensatory mechanisms linked to hippocampal neuron survival (KIF1B, YAP1). In the turquoise module seven out of its nine $\mathrm{HH}$ genes are positively correlated with the mild phenotype GCL0-1 (Fig. 5). Two HH genes in this module, $P I K 3 C 3$ and $D C P 1 B$, are respectively involved in CNS neuronal homeostasis and in regulating human memory performance, thus indicating protective and compensatory mechanisms. Significantly, all the genes mentioned above, excepting SPOCK1, are HHubs or eHubs i.e., genes with high intramodular and/or whole network connectivity. These genes probably are essential genes with a relevant role in defining the modules' biological function ${ }^{23,25}$, namely, the association of blue and turquoise modules with severe and mild phenotypes, respectively.

The red module, occupying a separate branch in the eigengene dendrogram, is highly and positively correlated with E-onset, and positively correlated with severe memory impairment (Fig. 2c). Early MTLE onset with HS has a large impact on brain plasticity and on brain connectivity and memory ${ }^{78,79}$. Moreover, early seizure onset is also a predictive factor for pharmacoresistancy ${ }^{6,80}$, being associated with a more severe functional abnormality in the ictal hippocampus ${ }^{79}$. Interestingly, five out of the six $\mathrm{HH}$ genes in the red module are involved in processes compatible with early tissue damage and subsequent microstructural reorganization, specifically: axon sprouting (OLFM1); neuronal circuitry remodeling (ST8SIA3); hippocampal neurogenesis, (NCDN and ERBB3), and astrocyte morphology remodeling (SYNJ2). Interestingly, the two hypo-expressed HH genes in the red-module, ERBB3 and SYNJ2, are also related to cognitive abilities and memory. Therefore, the co-expression profile, functional role, and 
connective hierarchy of the $\mathrm{HH}$ genes in the red module (Fig. 5) appear to be compatible with the module's phenotypic profile.

Contrariwise to hubs, that occupy a topologically central position in the modules and confer robustness to the co-expression network, HGS genes are usually at the network's periphery and their expression shows significant variation across trait groups ${ }^{23,25,81}$. The HGS genes are significant for the traits, as described in the Results section and depicted in Fig. 6, and here we show how these genes may serve as biomarkers or therapeutic targets for RMTLE.

Let us consider first the six HGS genes correlated with GCL. We found that ISYNA1, whose brain expression is persistently elevated in a rat model of epilepsy ${ }^{64}$, is more expressed in GCL3 than in GCL2 and GCL0-1, confirming its proposed role as RMTLE biomarker and its potential as a therapeutic target ${ }^{64,82}$. CHSY1 is related to the maintenance of hippocampal volume in RMTLE patients and its expression increases from GCL3 to GCL0-1, so indicating a compensatory mechanism and the gene's usefulness as biomarker. The genes AMFR and PTLP are respectively involved in neuronal survival and in neuroprotection, and both are necessary for memory and learning, but the expression of AMFR increases from GCL3 to GCL0-1 whereas that of PTLP decreases, thus indicating pathogenic and compensatory mechanisms. Finally, LGR4 and MGAT2, also HH genes and respectively involved in regulating $\mathrm{Wnt} / \beta$ catenin signaling and neuronal viability, have increased expression levels from GCL3 to GCL0-1, implying a compensatory mechanism and that these two genes may be meaningful biomarkers for RMTLE and hippocampal sclerosis.

Four HGS genes are correlated with GCD and two of them, ERBB4 and $M D H 2$, directly involved in epileptic processes, have significantly higher expression in GCD3 and GCD2, being potential biomarker candidates. The other two genes, SMARCAD1 and DHX9, are involved in chromatin remodeling and maintenance of genomic stability, and their average expression levels varies across GCD grades. The HGS gene HYOU1 has a neuroprotective role and, as expectable, is relatively higher expressed in the absence of GCB. $A S N S D 1$, a gene involved in biological processes related to memory, has a diminished expression in patients with severe memory impairment. Finally, three HGS genes, VLDR, FGFR2, and HBEGF, that are involved in synaptic plasticity and memory formation and belong to the red module - which is correlated with E-onset and memory impairment - have significant decreased expression in patients with early disease onset.

In conclusion, by adopting a systems biology approach and integrating clinical, histopathological and transcriptomic data, we were able to identify transcriptional modules highly correlated with DG alterations, cognitive dysfunctions, and disease onset in our RMTLE patients. The functional characterization of the high-hierarchy genes in each module allowed us to unveil the modules' main biological functions, paving the way for further investigations on their roles in RMTLE pathophysiology. Moreover, we found several HGS genes which have the potential to become novel biomarkers and/or therapeutic targets in RMTLE. These results are relevant considering the urge for identifying the genomic mechanisms underlying RMTLE, what could lead to more effective therapeutic interventions. 


\section{Material And Methods}

\section{Patients and brain tissue specimens}

A total of 57 RMTLE patients (aged 18-55 years) with unilateral mesial temporal sclerosis (MTS) who underwent corticoamygdalohippocampectomy were included in this study, which was approved by the research ethics committees of Hospital das Clínicas da FMUSP and Hospital Albert Einstein, São Paulo, SP, Brazil, under numbers 251/05 and CAEE 0122.0.028.174.05. A written informed consent was obtained from all patients. Refractory epilepsy cases were defined as those who have not gained seizure control after treatment with two or more antiepileptic drugs. None of the patients had intellectual disability or first-degree family members with epilepsy or febrile seizure history. All patients were diagnosed according to ILAE criteria ${ }^{83}$ and underwent preoperative clinical, electrophysiological, neuropsychological and neuroimaging evaluations. Tissue explants from CA3 were obtained at surgery room and immediately processed for global gene expression analysis. All the resected hippocampal specimens were analyzed by histopathology. The demographic, clinical, histological and cognitive data of all patients are summarized in Supplementary Table S1 online. All methods and experiments were carried out in accordance with relevant guidelines and regulations.

\section{Cognitive function evaluation}

A total of 44 patients and 60 healthy volunteers (Supplementary Table S2 online) were submitted to cognitive function evaluation. Patients and controls with IQ score $>75$, at least primary school education, and without psychiatric comorbidities, underwent a cognitive test battery that included: i) Rey auditory verbal (RAVLT) and visual design (RVDLT) learning test, Rey-Osterrieth complex figure test (ROCF), facial recognition, and logical memory, for memory; ii) semantic and phonological fluency, word comprehension, and object, verbal, proper noun and responsive naming; and iii) Stroop test (mistake and correction); Digit Span test, verbal fluency (FAS test), and Wisconsin test (WCST), for executive function (Supplementary Table S3 online). The collected data were analyzed using the Receiver Operating Curve (ROC) analysis, comparing patients' and controls' performances to yield best sensitivity and specificity, in order to establish a cut-off score for each test (Supplementary Table S3 online). We classified patients as normal or impaired, compared with controls, using Mann-Whitney test for each test. Values of $p<0.05$ were considered as statistically significant. We generated a memory, language and executive function impairment score. This score was based on failed test number.

\section{Histological analysis}

Histological analysis was performed in 43 resected hippocampi (Supplementary Table S1 online). Histological processing was undertaken as previously described ${ }^{84,85}$. Briefly, formalin-fixed sclerotic hippocampi were carefully oriented, trimmed and sectioned using a cryostat. One out of five coronal slices (sixty-micron thick) throughout the entire hippocampus length was mounted on gelatin-coated slices and processed for Nissl staining. 
The type of HS was described according to Blümcke et al. ${ }^{24}$ as HS ILAE type 1 (severe CA1 and CA4 neuronal loss and gliosis), HS ILAE type 2 (CA1 neuronal loss and gliosis), HS ILAE type 3 (CA4 neuronal loss and gliosis) or no-HS (preserved cell density and gliosis only), based on light microscopy of the hippocampal subfields, using a 5x objective lens (DM750, Leica Microsystems, Germany) throughout the entire hippocampus. Only sections presenting the three CA subfields were included.

Semiquantitative assessment focused on dentate gyrus abnormalities, particularly cytoarchitectural disorganization (loss, dispersion and bilamination) in four non-adjacent hippocampal body slices. Granule cell loss (GCL) was defined as areas of reduced granule cell layer width, reduced neuronal cell density or granule cell layer disruption. GCL was graded in three categories: grade zero to 1 - no GCL to mild neuronal density reduction, grade 2 - moderate neuronal density reduction, grade 3 - severe neuronal density reduction. Granule cell dispersion (GCD) was defined as broadening of the granule cell layer and loss of normal boundaries. GCD was graded into four subjective categories: grade zero - no GCD, grade 1 mild granule cell layer broadening with few ectopic granule cells in the inner molecular layer, grade 2 moderate granule cell layer broadening with ectopic granule cells beyond the inner molecular layer, and grade 3 - extensive granule cell layer broadening invading the outer molecular cell layer. Granule cell bilamination (GCB) was described as present or absent. Both ectopic clusters of granule cells in the molecular layer (focal GCB) and complete duplication of granule cell layer (diffuse GCB) were considered. Due to possible artifacts, we did not evaluate areas at angles, end points of the C-shaped granule cell layer, or blood vessels vicinities.

\section{RNA extraction}

Fresh explants from hippocampal CA3 region (3-4 $\mathrm{mm}^{3}$ ) from 22 RMTLE patients (Supplementary Table S1 online) were obtained at the surgery room and immediately preserved with RNAlater (Qiagen cat. no. 76106, Valencia, CA). Neuropathology analysis of the resected hippocampi confirmed that the CA3 explants were obtained at the proper site ${ }^{85}$. The preserved explants were homogenized with TissueRupter (Qiagen, cat. no. 9001272 Valencia, CA) and total RNA was extracted from the homogenates using the RNeasy Lipid Tissue Kit (Qiagen cat. no. 74804, Valencia, CA) according to the manufacturer's instructions. RNA quality was assessed on the Agilent BioAnalyzer 2100 (Agilent, Santa Clara, CA). All samples were stored at $-80^{\circ} \mathrm{C}$ until used in hybridization experiments.

\section{Microarray hybridization and gene expression analysis}

In order to determine gene expression profiles, 4x44K DNA microarrays (Whole Human Genome Microarray Kit, Agilent Technologies, cat no. G4112F, Santa Clara, CA) were used. The procedures for hybridization using the fluorescent dye Cy3 followed the manufacturer's protocols (One-Color MicroarrayBased Gene Expression Analysis - Quick Amp Labeling). The images were captured by the reader Agilent Bundle according to the parameters recommended for bioarrays and extracted by Agilent Feature Extraction software version 9.5.3. Spots with two or more flags (low intensity, saturation, controls, etc.) were considered as NA, that is, without valid expression value. An in-house algorithm in R environment (version $3.4 .4^{86}$ ) was used for excluding transcript spots presenting one or more NAs and for converting 
gene expression values to log base 2. Through this procedure we identified 8,104 Gene Ontology (GO) annotated genes. Data normalization was performed using limma package ${ }^{87,88}$ in R environment (version $\left.3.4 .4^{86}\right)$. All microarray raw data have been deposited in GEO public database (http://www.ncbi.nlm.nih.gov/geo), a MIAME compliant database, under accession number GSE163296.

\section{Weighted Gene Co-expression Network Analysis (WGCNA)}

The normalized gene expression data was used for WGCNA. Network was constructed using the WGCNA package ${ }^{22}$ in R environment (version 3.4.4 ${ }^{86}$ ). Pearson's correlation coefficient was used for obtaining gene co-expression similarity measures and for the subsequent construction of an adjacency matrix using soft power and topological overlap matrix (TOM). Soft-thresholding process transforms the correlation matrix to mimic the scale-free topology. TOM is used to filter weak connections during network construction. Module identification is based on TOM and in average linkage hierarchical clustering. Keeping to the scale-free topology criterion, soft power $\beta=12\left(R^{2}=0.910\right)$ was considered (Supplementary Fig. S5 online). Finally, dynamic tree cut algorithm was used for dendrogram's branch selection. The module eigengene (ME) is defined as the first principal component of a given module, which can be considered a representative of the gene expression profiles in a module. Module Membership (MM), also known as eigengene-based connectivity (kME), is defined as the correlation of each gene expression profile with the module eigengene of a given module.

\section{Module-trait association}

Firstly, we obtained the gene significance (GS), i.e. a value for the correlation between specific traits (Supplementary Table S1 online) and gene expression profiles ${ }^{22}$. This analysis was conducted for three different trait matrices. One matrix for demographic, clinical, and histopathological data of the 22 TLE patients and the two other matrices for cognitive features: i) one for the left HS patients $(n=14)$, and ii) another for right HS patients $(n=8)$. The average GS value for a particular module is considered as a measure of module significance (MS). The GS values were obtained using Pearson's correlation and, for assigning a p-value to the module significance, we used the Student's $t$ test. The modules presenting high positive or negative correlation values $(r \geq|0.50|$ and $p<0.05)$ with a trait were selected for functional analysis. Modular gene set enrichment analysis for GO Biological Process (BP) terms was accomplished by using the Enrichr online web-based tool ${ }^{26}$.

\section{Node categorization}

Intramodular node connectivity was calculated considering: i) $k_{\text {Total, }}$, the whole network connectivity of each gene; ii) $k_{\text {Within, }}$ gene connections with other genes in the same module ${ }^{22}$. Genes presenting high $k_{\text {Total }}$ and $k_{\text {Within }}$ are classified as high hubs (HHubs), genes presenting high $k_{\text {Total }}$ but low $k_{\text {Within }}$ are called eHubs, and genes presenting high $k_{\text {Within }}$ but low $k k_{\text {Total }}$ are the iHubs. We plotted all gene values in a $k_{\text {Total }}$ vs. $k_{\text {Within }}$ graphic (Supplementary Figs. S3 and S4 online). Additionally, the differential gene expression profile was assessed trough GS values, ie. the GS of the $n^{\text {th }}$ gene is the correlation measure 
between the $n^{\text {th }}$ gene expression and the specific trait. Then, when the GS value is positive or negative it means that the $n^{\text {th }}$ gene is hyper- or hypo-expressed for the specific trait.

\section{Genes presenting high gene significance (GS) values (HGS genes)}

The Module Membership (MM), i.e. a measure of intramodular connectivity ${ }^{22}$, and the GS values were used for candidate gene marker identification. Genes presenting high GS and high MM were considered as HGS genes in the module and significantly associated with at least one trait. This analysis was conducted for left and right sides separately. We plotted all gene values in a MM ( $x$-axis) vs. GS graphic (y-axis).

\section{Clinical and pathological correlation analyses}

Clinical and pathological data from 43 patients and respective hippocampal samples (Supplementary Table S1 online) were used for correlation analyses between a pair of selected phenotypic variables, e.g. GCL grade vs. memory impairment score for left-HS patients, IPI vs. HS side, HS vs. language impairment, etc. Comparison groups were set for three data matrices: i) including all patients; ii) LHS group, including left-HS patients only; and iii) RHS group, including right HS patients only. These analyses were conducted using the Spearman test and the $p$ value $<0.05$ was considered significant.

\section{Declarations}

\section{Acknowledgments}

We would like to thank Leandro Rodrigues Ferreira, Nara Carpinelli, Viviane Yumi Hatano, Marizia Toma, Paula Santos and Fernando Rosa for technical support; Alexandre Valotta da Silva for histopathological analysis; Bettina Castro, Cristiane Messas, Carla Cristina Adda, Juliana Passos de Almeida for the application of the cognitive tests. This work was funded by Fundação de Amparo à Pesquisa do Estado de São Paulo (FAPESP) grants no. 2005/56446-0 (CInAPCe Program) and 2015/22308-2, and by Conselho Nacional de Desenvolvimento Científico e Tecnológico (CNPq) grant no. 306893/2018-5 to C.A.M-F.

\section{Author Contributions}

Conceived and designed the experiments: S.Y.B., F.B.B., C.A.M-F., L.H.M.C., L.R.P-S. Performed the experiments: S.Y.B., F.B.B., L.R.P-S., J.G.M.O., M.A.D.C., M.H.M.O. Analyzed the data: S.Y.B., F.B.B., L.R.P-S., J.G.M.O., M.A.D.C., M.H.M.O. Funding acquisition: C.A.M-F. Resources: L.H.M.C., H-T.W, C.A.M-F. Wrote the paper: S.Y.B., F.B.B., C.A.M-F., L.R.P-S., L.H.M.C.

\section{Additional Information}

Supplementary information accompanies this paper. 


\section{Competing Interests}

The authors declare no competing interests.

\section{Data Availability Statement}

The datasets generated during and/or analyzed during the current study are available from the corresponding author on reasonable request.

\section{References}

1. Beghi, E. The Epidemiology of Epilepsy. Neuroepidemiology. 54, 185-191 https://doi.org/10.1159/000503831 (2020).

2. Malmgren, K. \& Thom, M. Hippocampal sclerosis-origins and imaging. Epilepsia. 53 (Suppl 4), 1933 https://doi.org/10.1111/j.1528-1167.2012.03610.x (2012).

3. Baulac, M. MTLE with hippocampal sclerosis in adult as a syndrome. Rev Neurol (Paris). 171, 259266 https://doi.org/10.1016/j.neurol.2015.02.004 (2015).

4. Chungath, M. \& Shorvon, S. The mortality and morbidity of febrile seizures. Nat Clin Pract Neurol. 4, 610-621 https://doi.org/10.1038/ncpneuro0922 (2008).

5. Blümcke, I. et al. A new clinico-pathological classification system for mesial temporal sclerosis. Acta Neuropathol. 113, 235-244 https://doi.org/10.1007/s00401-006-0187-0 (2007).

6. Sànchez, J., Centanaro, M., Solís, J., Delgado, F. \& Yépez, L. Factors predicting the outcome following medical treatment of mesial temporal epilepsy with hippocampal sclerosis. Seizure. 23, 448-453 https://doi.org/10.1016/j.seizure.2014.03.003 (2014).

7. Lee, S. K. Treatment strategy for the patient with hippocampal sclerosis who failed to the first antiepileptic drug. J Epilepsy Res. 4, 1-6 (2014).

8. Engel, J. Approaches to refractory epilepsy. Ann Indian Acad Neurol. 17, S12-17 https://doi.org/10.4103/0972-2327.128644 (2014).

9. Seki, T., Hori, T., Miyata, H., Maehara, M. \& Namba, T. Analysis of proliferating neuronal progenitors and immature neurons in the human hippocampus surgically removed from control and epileptic patients. Sci Rep. 9, 18194 https://doi.org/10.1038/s41598-019-54684-z (2019).

10. Blumcke, I. et al. Histopathological Findings in Brain Tissue Obtained during Epilepsy Surgery. N Engl J Med. 377, 1648-1656 https://doi.org/10.1056/NEJMoa1703784 (2017).

11. Liu, J. Y. W. et al. Granule Cell Dispersion in Human Temporal Lobe Epilepsy: Proteomics Investigation of Neurodevelopmental Migratory Pathways. Front Cell Neurosci. 14, 53 https://doi.org/10.3389/fncel.2020.00053 (2020).

12. Krook-Magnuson, E. et al. In vivo evaluation of the dentate gate theory in epilepsy. J Physiol. 593, 2379-2388 https://doi.org/10.1113/JP270056 (2015). 
13. Queenan, B. N. et al. Kappa opioid receptors regulate hippocampal synaptic homeostasis and epileptogenesis. Epilepsia. 59, 106-122 https://doi.org/10.1111/epi.13941 (2018).

14. Canto, A. M. et al. Multi-omics analysis suggests enhanced epileptogenesis in the Cornu Ammonis 3 of the pilocarpine model of mesial temporal lobe epilepsy. Hippocampus. https://doi.org/10.1002/hipo.23268 (2020).

15. Winden, K. D. et al. A systems level, functional genomics analysis of chronic epilepsy. PLoS One. 6 , e20763 https://doi.org/10.1371/journal.pone.0020763 (2011).

16. Bando, S. Y. et al. Complex network analysis of CA3 transcriptome reveals pathogenic and compensatory pathways in refractory temporal lobe epilepsy. PLoS One. 8, e79913 https://doi.org/10.1371/journal.pone.0079913 (2013).

17. Moreira-Filho, C. A. et al. Community structure analysis of transcriptional networks reveals distinct molecular pathways for early- and late-onset temporal lobe epilepsy with childhood febrile seizures. PLoS One. 10, e0128174 https://doi.org/10.1371/journal.pone.0128174 (2015).

18. Guelfi, S. et al. Transcriptomic and genetic analyses reveal potential causal drivers for intractable partial epilepsy. Brain. 142, 1616-1630 https://doi.org/10.1093/brain/awz074 (2019).

19. Li, Y., Wang, C., Wang, P., Li, X. \& Zhou, L. Effects of febrile seizures in mesial temporal lobe epilepsy with hippocampal sclerosis on gene expression using bioinformatical analysis. Acta Epileptologica. 2, https://doi.org/10.1186/s42494-020-00027-9 (2020).

20. Engel, J. et al. Early surgical therapy for drug-resistant temporal lobe epilepsy: a randomized trial. JAMA. 307, 922-930 https://doi.org/10.1001/jama.2012.220 (2012).

21. Margineanu, D. G. Systems biology, complexity, and the impact on antiepileptic drug discovery. Epilepsy Behav. 38, 131-142 https://doi.org/10.1016/j.yebeh.2013.08.029 (2014).

22. Langfelder, P. \& Horvath, S. WGCNA: an R package for weighted correlation network analysis. $B M C$ Bioinformatics. 9, 559 https://doi.org/10.1186/1471-2105-9-559 (2008).

23. Barabási, A. L., Gulbahce, N. \& Loscalzo, J. Network medicine: a network-based approach to human disease. Nat Rev Genet. 12, 56-68 https://doi.org/10.1038/nrg2918 (2011).

24. Blümcke, I. et al. International consensus classification of hippocampal sclerosis in temporal lobe epilepsy: a Task Force report from the ILAE Commission on Diagnostic Methods. Epilepsia. 54, 1315-1329 https://doi.org/10.1111/epi.12220 (2013).

25. van Dam, S., Võsa, U., van der Graaf, A., Franke, L. \& de Magalhães, J. P. Gene co-expression analysis for functional classification and gene-disease predictions. Brief Bioinform. 19, 575-592 https://doi.org/10.1093/bib/bbw139 (2018).

26. Kuleshov, M. V. et al. Enrichr: a comprehensive gene set enrichment analysis web server 2016 update. Nucleic Acids Res. 44, W90-97 https://doi.org/10.1093/nar/gkw377 (2016).

27. Langfelder, P., Zhang, B. \& Horvath, S. Dynamic Tree Cut: in-depth description, tests and applications., (2007). 
28. Hirota, $\mathrm{S}$. et al. Neuropilin 1 balances $\beta 8$ integrin-activated TGF $\beta$ signaling to control sprouting angiogenesis in the brain. Development. 142, 4363-4373 https://doi.org/10.1242/dev.113746 (2015).

29. Chang, J. et al. A mutation in the mitochondrial protein UQCRB promotes angiogenesis through the generation of mitochondrial reactive oxygen species. Biochem Biophys Res Commun. 455, 290-297 https://doi.org/10.1016/j.bbrc.2014.11.005 (2014).

30. Marchi, N. \& Lerner-Natoli, M. Cerebrovascular remodeling and epilepsy. Neuroscientist. 19, 304-312 https://doi.org/10.1177/1073858412462747 (2013).

31. Xu, F. et al. KIF1B $\beta$ mutations detected in hereditary neuropathy impair IGF1R transport and axon growth. J Cell Biol. 217, 3480-3496 https://doi.org/10.1083/jcb.201801085 (2018).

32. Li, X., Yao, L., Liang, Q., Qu, H. \& Cai, H. Propofol Protects Hippocampal Neurons from HypoxiaReoxygenation Injury by Decreasing Calcineurin-Induced Calcium Overload and Activating YAP Signaling. Oxid Med Cell Longev 2018, 1725191, doi:10.1155/2018/1725191 (2018).

33. Gasparini, A., Tosatto, S. C. E., Murgia, A. \& Leonardi, E. Dynamic scaffolds for neuronal signaling: in silico analysis of the TANC protein family. Sci Rep. 7, 6829 https://doi.org/10.1038/s41598-01705748-5 (2017).

34. Fiermonte, G. et al. Identification of the mitochondrial glutamate transporter. Bacterial expression, reconstitution, functional characterization, and tissue distribution of two human isoforms. J Biol Chem. 277, 19289-19294 https://doi.org/10.1074/jbc.M201572200 (2002).

35. Vandenberg, R. J. \& Ryan, R. M. Mechanisms of glutamate transport. Physiol Rev. 93, 1621-1657 https://doi.org/10.1152/physrev.00007.2013 (2013).

36. Iseki, K. et al. Altered expression pattern of testican-1 mRNA after brain injury. Biomed Res. 32, 373378 https://doi.org/10.2220/biomedres.32.373 (2011).

37. Kimura, I. et al. Neuferricin, a novel extracellular heme-binding protein, promotes neurogenesis. $J$ Neurochem. 112, 1156-1167 https://doi.org/10.1111/j.1471-4159.2009.06522.x (2010).

38. Ko, Y. U. et al. Site-specific phosphorylation of Fbxw7 by Cdk5/p25 and its resulting decreased stability are linked to glutamate-induced excitotoxicity. Cell Death Dis. 10, 579 https://doi.org/10.1038/s41419-019-1818-4 (2019).

39. Haile, Y. et al. Rab32 connects ER stress to mitochondrial defects in multiple sclerosis. $J$ Neuroinflammation. 14, 19 https://doi.org/10.1186/s12974-016-0788-z (2017).

40. Wang, L., Budolfson, K. \& Wang, F. Pik3c3 deletion in pyramidal neurons results in loss of synapses, extensive gliosis and progressive neurodegeneration. Neuroscience. 172, 427-442 https://doi.org/10.1016/j.neuroscience.2010.10.035 (2011).

41. Lorenzo, D. N. et al. A PIK3C3-ankyrin-B-dynactin pathway promotes axonal growth and multiorganelle transport. J Cell Biol. 207, 735-752 https://doi.org/10.1083/jcb.201407063 (2014).

42. Schröder, J. et al. MicroRNA-138 is a potential regulator of memory performance in humans. Front Hum Neurosci. 8, 501 https://doi.org/10.3389/fnhum.2014.00501 (2014). 
43. Raslan, A. A. \& Yoon, J. K. R-spondins: Multi-mode WNT signaling regulators in adult stem cells. Int J Biochem Cell Biol. 106, 26-34 https://doi.org/10.1016/j.biocel.2018.11.005 (2019).

44. Lee, D. Y. Roles of mTOR Signaling in Brain Development. Exp Neurobiol. 24, 177-185 https://doi.org/10.5607/en.2015.24.3.177 (2015).

45. Huang, C., Fu, X. H., Zhou, D. \& Li, J. M. The Role of Wnt/ $\beta$-Catenin Signaling Pathway in Disrupted Hippocampal Neurogenesis of Temporal Lobe Epilepsy: A Potential Therapeutic Target?. Neurochem Res. 40, 1319-1332 https://doi.org/10.1007/s11064-015-1614-1 (2015).

46. Sha, L. Z. et al. Mapping the spatio-temporal pattern of the mammalian target of rapamycin (mTOR) activation in temporal lobe epilepsy. PLoS One. 7, e39152 https://doi.org/10.1371/journal.pone.0039152 (2012).

47. Kim, J. K. et al. Brain somatic mutations in MTOR reveal translational dysregulations underlying intractable focal epilepsy. J Clin Invest. 129, 4207-4223 https://doi.org/10.1172/JCl127032 (2019).

48. Tan, J., Dunn, J., Jaeken, J. \& Schachter, H. Mutations in the MGAT2 gene controlling complex Nglycan synthesis cause carbohydrate-deficient glycoprotein syndrome type II, an autosomal recessive disease with defective brain development. Am J Hum Genet. 59, 810-817 (1996).

49. Tang, H. et al. Architecture of cell-cell adhesion mediated by sidekicks. Proc Natl Acad Sci U S A. 115, 9246-9251 https://doi.org/10.1073/pnas.1801810115 (2018).

50. Nakaya, N., Sultana, A., Lee, H. S. \& Tomarev, S. I. Olfactomedin 1 interacts with the Nogo A receptor complex to regulate axon growth. J Biol Chem. 287, 37171-37184 https://doi.org/10.1074/jbc.M112.389916 (2012).

51. Lin, C. Y. et al. Functional roles of ST8SIA3-mediated sialylation of striatal dopamine D. Trans/ Psychiatry. 9, 209 https://doi.org/10.1038/s41398-019-0529-z (2019).

52. Mikkonen, M. et al. Remodeling of neuronal circuitries in human temporal lobe epilepsy: increased expression of highly polysialylated neural cell adhesion molecule in the hippocampus and the entorhinal cortex. Ann Neurol. 44, 923-934 https://doi.org/10.1002/ana.410440611 (1998).

53. Janz, R., Hofmann, K. \& Südhof, T. C. SVOP, an evolutionarily conserved synaptic vesicle protein, suggests novel transport functions of synaptic vesicles. J Neurosci. 18, 9269-9281 (1998).

54. Bartholome, O. et al. Puzzling Out Synaptic Vesicle 2 Family Members Functions. Front Mol Neurosci. 10, 148 https://doi.org/10.3389/fnmol.2017.00148 (2017).

55. Xu, Y. et al. Altered Norbin Expression in Patients with Epilepsy and a Rat Model. Sci Rep. 7, 13970 https://doi.org/10.1038/s41598-017-13248-9 (2017).

56. Mahar, I. et al. Subchronic peripheral neuregulin-1 increases ventral hippocampal neurogenesis and induces antidepressant-like effects. PLoS One. 6, e26610 https://doi.org/10.1371/journal.pone.0026610 (2011).

57. Bellesi, M., de Vivo, L., Tononi, G. \& Cirelli, C. Effects of sleep and wake on astrocytes: clues from molecular and ultrastructural studies. BMC Biol. 13, 66 https://doi.org/10.1186/s12915-015-0176-7 (2015). 
58. Lopez, L. M. et al. Evolutionary conserved longevity genes and human cognitive abilities in elderly cohorts. Eur J Hum Genet. 20, 341-347 https://doi.org/10.1038/ejhg.2011.201 (2012).

59. Leong, H. S. \& Kipling, D. Text-based over-representation analysis of microarray gene lists with annotation bias. Nucleic Acids Res. 37, e79 https://doi.org/10.1093/nar/gkp310 (2009).

60. Luo, Y. et al. A link between maze learning and hippocampal expression of neuroleukin and its receptor gp78. J Neurochem. 80, 354-361 https://doi.org/10.1046/j.0022-3042.2001.00707.x (2002).

61. Yang, Y., Cheng, X. R., Zhang, G. R., Zhou, W. X. \& Zhang, Y. X. Autocrine motility factor receptor is involved in the process of learning and memory in the central nervous system. Behav Brain Res. 229, 412-418 https://doi.org/10.1016/j.bbr.2012.01.043 (2012).

62. Wang, H. et al. PLTP deficiency impairs learning and memory capabilities partially due to alteration of amyloid- $\beta$ metabolism in old mice. J Alzheimers Dis. 39, 79-88 https://doi.org/10.3233/JAD130812 (2014).

63. Peixoto-Santos, J. E. et al. Temporal lobe epilepsy patients with severe hippocampal neuron loss but normal hippocampal volume: Extracellular matrix molecules are important for the maintenance of hippocampal volume. Epilepsia. 56, 1562-1570 https://doi.org/10.1111/epi.13082 (2015).

64. Pascente, R. et al. Cognitive deficits and brain myo-Inositol are early biomarkers of epileptogenesis in a rat model of epilepsy. Neurobiol Dis. 93, 146-155 https://doi.org/10.1016/j.nbd.2016.05.001 (2016).

65. Pimentel-Silva, L. R. et al. Interactions between in vivo neuronal-glial markers, side of hippocampal sclerosis, and pharmacoresponse in temporal lobe epilepsy. Epilepsia. 61, 1008-1018 https://doi.org/10.1111/epi.16509 (2020).

66. Hodges, S. L. \& Lugo, J. N. Wnt/ $\beta$-catenin signaling as a potential target for novel epilepsy therapies. Epilepsy Res. 146, 9-16 https://doi.org/10.1016/j.eplepsyres.2018.07.002 (2018).

67. Chen, Y. H. et al. MDH2 is an RNA binding protein involved in downregulation of sodium channel Scn1a expression under seizure condition. Biochim Biophys Acta Mol Basis Dis. 1863, 1492-1499 https://doi.org/10.1016/j.bbadis.2017.04.018 (2017).

68. Kaphzan, H. et al. Reversal of impaired hippocampal long-term potentiation and contextual fear memory deficits in Angelman syndrome model mice by ErbB inhibitors. Biol Psychiatry. 72, 182-190 https://doi.org/10.1016/j.biopsych.2012.01.021 (2012).

69. Zhu, J. M. et al. Increased NRG1-ErbB4 signaling in human symptomatic epilepsy. Sci Rep. 7, 141 https://doi.org/10.1038/s41598-017-00207-7 (2017).

70. Lim, D. A. et al. In vivo transcriptional profile analysis reveals RNA splicing and chromatin remodeling as prominent processes for adult neurogenesis. Mol Cell Neurosci. 31, 131-148 https://doi.org/10.1016/j.mcn.2005.10.005 (2006).

71. Lee, T. \& Pelletier, J. The biology of DHX9 and its potential as a therapeutic target. Oncotarget. 7, 42716-42739 https://doi.org/10.18632/oncotarget.8446 (2016). 
72. Zhao, L., Rosales, C., Seburn, K., Ron, D. \& Ackerman, S. L. Alteration of the unfolded protein response modifies neurodegeneration in a mouse model of Marinesco-Sjögren syndrome. Hum Mol Genet. 19, 25-35 https://doi.org/10.1093/hmg/ddp464 (2010).

73. Zhu, X. et al. Neuronal Nitric Oxide Synthase Contributes to PTZ Kindling Epilepsy-Induced Hippocampal Endoplasmic Reticulum Stress and Oxidative Damage. Front Cell Neurosci. 11, 377 https://doi.org/10.3389/fncel.2017.00377 (2017).

74. Ruzzo, E. K. et al. Deficiency of asparagine synthetase causes congenital microcephaly and a progressive form of encephalopathy. Neuron. 80, 429-441 https://doi.org/10.1016/j.neuron.2013.08.013 (2013).

75. Jakob, B. et al. Intersectin 1 is a component of the Reelin pathway to regulate neuronal migration and synaptic plasticity in the hippocampus. Proc Natl Acad Sci U S A. 114, 5533-5538 https://doi.org/10.1073/pnas.1704447114 (2017).

76. Stevens, H. E., Jiang, G. Y., Schwartz, M. L. \& Vaccarino, F. M. Learning and memory depend on fibroblast growth factor receptor 2 functioning in hippocampus. Biol Psychiatry. 71, 1090-1098 https://doi.org/10.1016/j.biopsych.2012.03.013 (2012).

77. Oyagi, A. et al. Heparin-binding EGF-like growth factor is required for synaptic plasticity and memory formation. Brain Res. 1419, 97-104 https://doi.org/10.1016/j.brainres.2011.09.003 (2011).

78. Doucet, G., Osipowicz, K., Sharan, A., Sperling, M. R. \& Tracy, J. I. Extratemporal functional connectivity impairments at rest are related to memory performance in mesial temporal epilepsy. Hum Brain Mapp. 34, 2202-2216 https://doi.org/10.1002/hbm.22059 (2013).

79. Doucet, G. E. et al. Early and late age of seizure onset have a differential impact on brain restingstate organization in temporal lobe epilepsy. Brain Topogr. 28, 113-126 https://doi.org/10.1007/s10548-014-0366-6 (2015).

80. Bilevicius, E. et al. Antiepileptic drug response in temporal lobe epilepsy: a clinical and MRI morphometry study. Neurology. 75, 1695-1701 https://doi.org/10.1212/WNL.0b013e3181fc29dd (2010).

81. Bertonha, F. B. et al. Age-related transcriptional modules and TF-miRNA-mRNA interactions in neonatal and infant human thymus. PLoS One. 15, e0227547 https://doi.org/10.1371/journal.pone.0227547 (2020).

82. Frej, A. D., Otto, G. P. \& Williams, R. S. Tipping the scales: Lessons from simple model systems on inositol imbalance in neurological disorders. Eur J Cell Biol. 96, 154-163 https://doi.org/10.1016/j.ejcb.2017.01.007 (2017).

83. Scheffer, I. E. et al. ILAE classification of the epilepsies: Position paper of the ILAE Commission for Classification and Terminology. Epilepsia. 58, 512-521 https://doi.org/10.1111/epi.13709 (2017).

84. Silva, A. V. et al. Granular cell dispersion and bilamination: two distinct histopathological patterns in epileptic hippocampi?. Epileptic Disord. 9, 438-442 https://doi.org/10.1684/epd.2007.0135 (2007).

85. Bando, S. Y. et al. Hippocampal CA3 transcriptome signature correlates with initial precipitating injury in refractory mesial temporal lobe epilepsy. PLoS One. 6, e26268 
https://doi.org/10.1371/journal.pone.0026268 (2011).

86. Team, R. C. (R Foundation for Statistical Computing, Vienna, Austria, 2018).

87. Ritchie et al. Vol. 43 e47 (Nucleic Acids Research, 2015).

88. Smyth, G. Limma: linear models for microarray data. 397-420(Springer, 2005).

\section{Figures}

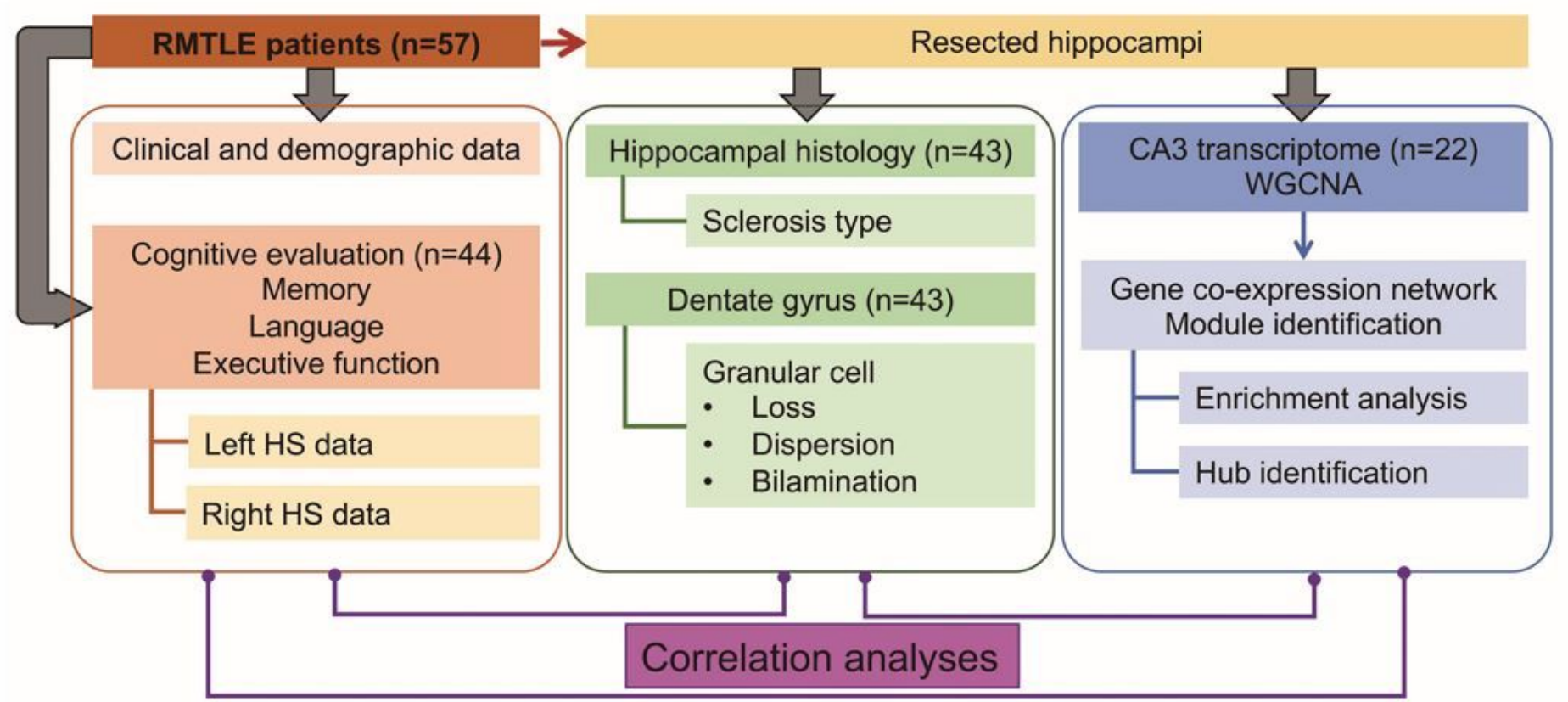

\section{Figure 1}

Study Workflow. Integrative analysis of demographic, clinical, histopathological, and genomic data. 
a

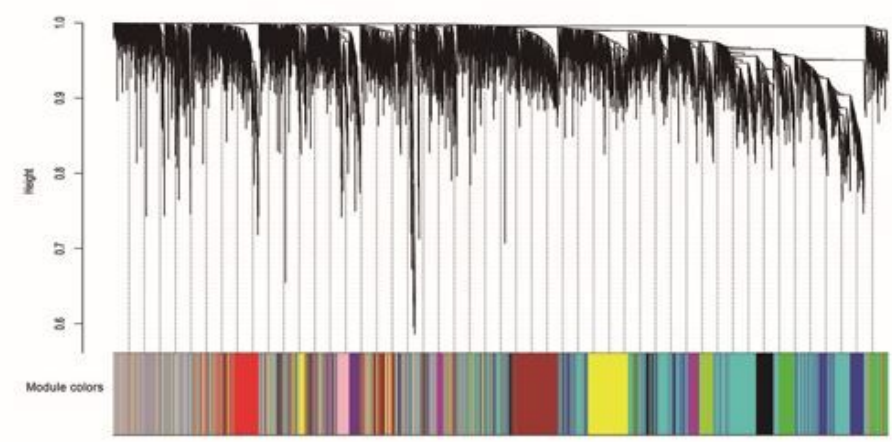

b

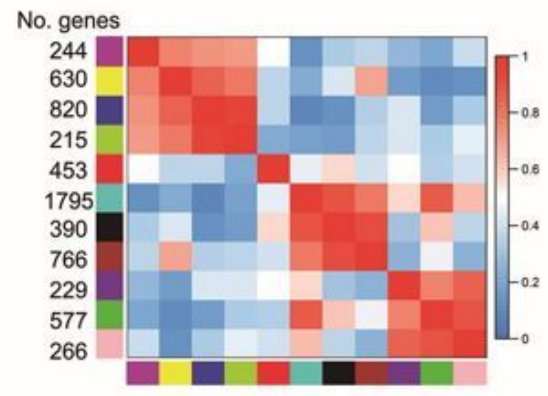

C

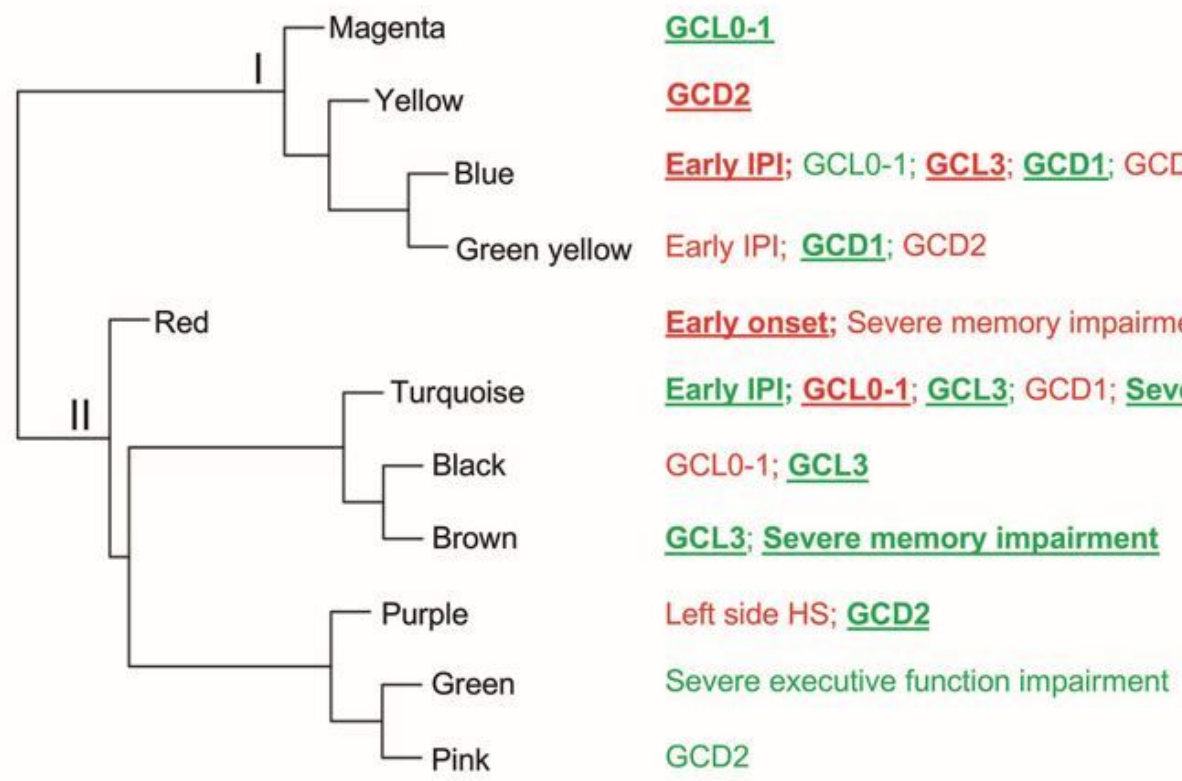

Figure 2

WGCNA analysis. Gene dendrogram and gene clustering analysis for module identification (a). Heatmap plot of the adjacencies in the eigengene network (b). Hierarchical clustering dendrogram of module eigengenes - evidencing the presence of two meta-modules (I and II) - and traits significantly correlated with modules (c). Positive or negative module-trait correlations depicted in red or green, respectively; the high module-trait correlations $(r>|0.50|)$ are indicated by bold underlined letters. 


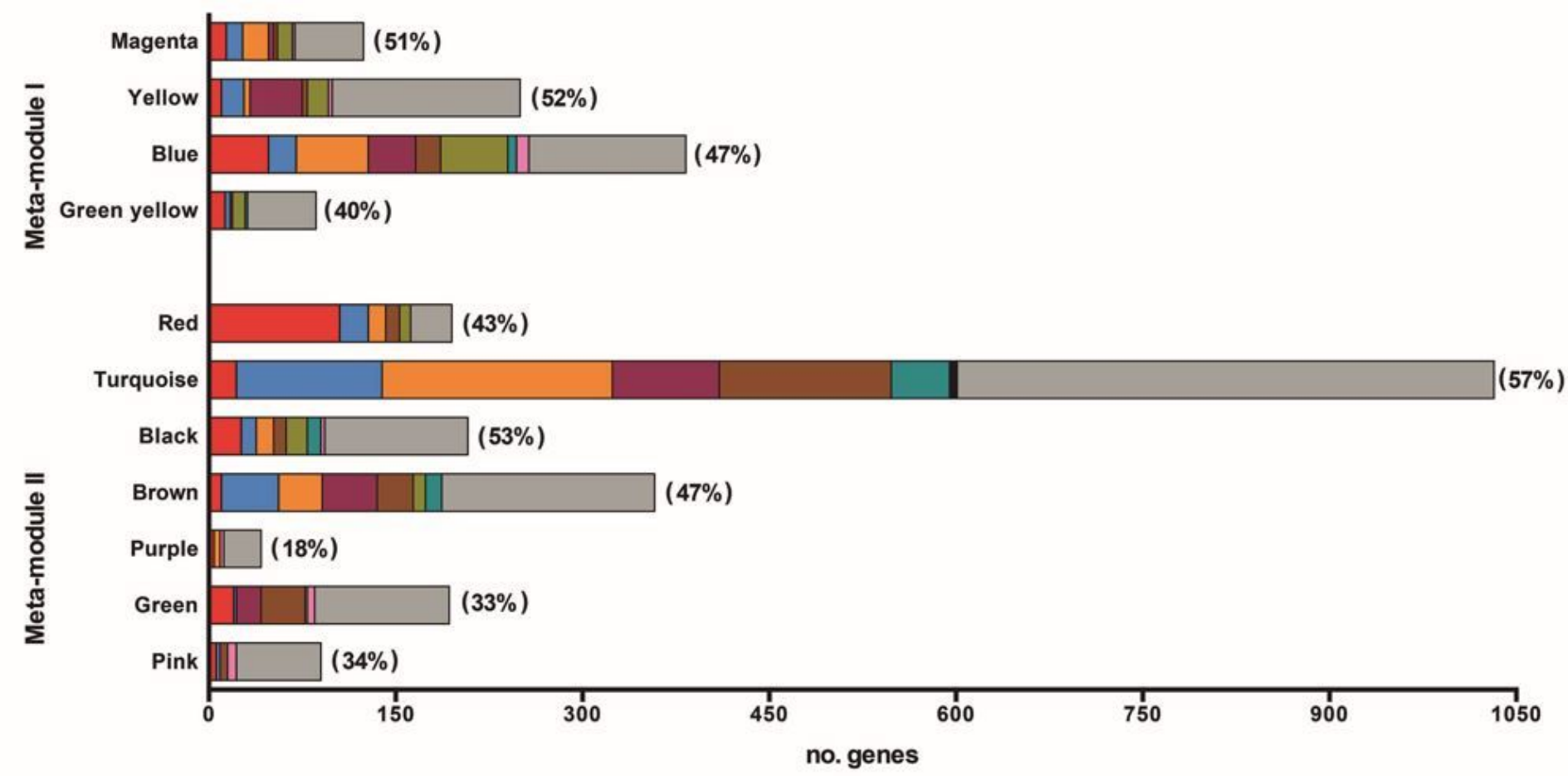

\footnotetext{
Molecular and cellular processes relevant to MTLE and brain functioning

neuron; glia; axon; myelination; excitability; synapse

Golgi-ER; endosome; exosome; molecule transport; ion transport

$\square$ signaling pathways; cytokines

ubiquitination

apoptosis; cell death

cytoskeleton; cell adhesion; cell migration and motility
}

Other related molecular and cellular processes

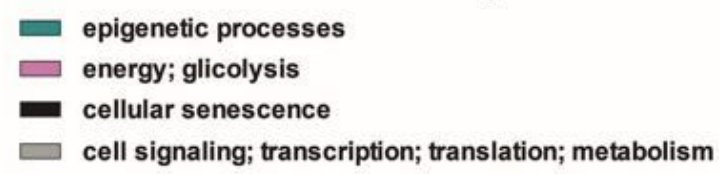

\section{Figure 3}

Histogram of enriched GO Biological Process (BP) terms for each WGCNA gene module. Different colors represent GO BP terms comprised in two main categories: i) molecular and cellular processes relevant to MTLE and brain functioning; ii) other related molecular and cellular processes. Between round brackets: percentage of genes over-represented in BP terms. 
Blue module

\begin{tabular}{|l|l|l|c|c|c|c|}
\hline Category & Gene & GO database: biological process; molecular function & E-IPI & GCL3 & GCD1 & SEFI \\
\hline \multirow{4}{*}{ HHub } & ITGB8 & positive regulation of angiogenesis & 0.61 & 0.64 & -0.58 \\
& UQCRB & oxidative phosphorylation & 0.60 & 0.60 & -0.53 \\
& KIF1B & anterograde axonal transport & 0.58 & 0.55 \\
& KIAA1143 & protein binding & 0.53 & 0.54 & -0.60 \\
& ZNF708 & regulation of transcription by RNA polymerase II & 0.55 & 0.61 & -0.50 \\
\hline \multirow{2}{*}{ iHub } & PDZD8 & mitochondrion-endoplasmic reticulum membrane tethering & 0.62 & -0.46 \\
& MAN1A2 & N-glycan processing & 0.60 & 0.51 \\
\hline \multirow{2}{*}{ eHub } & YAP1 & hippo signaling & 0.57 & 0.53 & -0.59 \\
& G3BP2 & stress granule assembly & 0.63 & 0.55 & -0.54 \\
\hline
\end{tabular}

\begin{tabular}{l} 
Yellow module \\
\begin{tabular}{|l|l|l|c|}
\hline Category & Gene & GO database: biological process; molecular function & GCD2 \\
\hline \multirow{2}{*}{ HHub } & CYB5D2 & positive regulation of neuron differentiation & -0.54 \\
& TANC2 & glutamatergic synapse & 0.63 \\
& SEC63 & post translational protein targeting to endoplasmic reticulum membrane & 0.57 \\
\hline \multirow{4}{*}{ iHub } & SPOCK1 & central nervous system neuron differentiation & 0.61 \\
& KRIT1 & negative regulation of angiogenesis & 0.54 \\
& ZZZ3 & regulation of transcription by RNA polymerase II & 0.67 \\
\hline \multirow{4}{*}{ eHub } & ZNF207 & regulation of transcription, DNA-templated & 0.60 \\
\hline & SLC25A18 & L-glutamate transmembrane transport & 0.52 \\
& ORC3L & origin recognition complex & 0.44 \\
& KCMF1 & protein ubiquitination & 0.47 \\
\hline
\end{tabular} \\
\hline
\end{tabular}

Green yellow module

\begin{tabular}{|l|l|l|c|}
\hline Category & Gene & GO database: biological process; molecular function & GCD1 \\
\hline HHub & FAM73A & mitochondrial fusion & -0.64 \\
\hline iHub & MPP5 & myelin assembly & -0.64 \\
& RFC1 & nucleotide-excision repair, DNA incision & -0.68 \\
\hline & FBXW7 & positive regulation of protein ubiquitination & 0.56 \\
& EIF2C2 & mRNA cleavage involved in gene silencing by miRNA & -0.56 \\
& PBX2 & positive regulation of transcription by RNA polymerase II & -0.50 \\
eHub & CUEDC1 & protein binding & -0.70 \\
& SP1 & regulation of transcription, DNA-templated & -0.54 \\
& KIAA0892 & maintenance of mitotic sister chromatid cohesion & -0.57 \\
& CHD9 & chromatin organization & -0.48 \\
\hline
\end{tabular}

\begin{tabular}{l} 
Magenta module \\
\begin{tabular}{|l|l|l|c|}
\hline Category & Gene & GO database: biological process; molecular function & GCL $0-1$ \\
\hline HHub & GNAI2 & positive regulation of neural precursor cell proliferation & -0.68 \\
& PLEKHB1 & regulation of cell differentiation & -0.64 \\
\hline \multirow{2}{*}{ iHub } & TUBG2 & microtubule nucleation & -0.64 \\
& MLF2 & regulation of transcription, DNA-templated & -0.61 \\
\hline \multirow{5}{*}{ eHub } & RAB32 & mitochondrion organization & 0.69 \\
& RNF5 & ubiquitin-protein transferase activity & -0.62 \\
& AKT1S1 & negative regulation of TOR signaling & -0.56 \\
& NUCB1 & calcium ion binding & -0.50 \\
& LGALS3BP & endocytosis & -0.71 \\
\hline
\end{tabular} \\
\cline { 2 - 3 }
\end{tabular}

\section{Figure 4}

High hierarchical genes (Hhubs, iHubs, and eHubs) of the meta-module I present in modules highly associated with at least one trait. Each $\mathrm{HH}$ gene is identified by its hierarchical categorization and $\mathrm{GO}$ biological process or molecular function. Columns in red or in green indicate genes with positive (i.e. hyper-expressed) or negative (i.e. hypo-expressed) GS values for a specific trait, respectively. NS indicates gene with non-significant $(p \geq 0.05)$ GS value for the specific trait. E-IPI stands for early IPI. SEFI stands for severe executive function impairment. The abbreviations - GCD1, GCD2, GCL0-1, GCL3, HS ILAE type 3 - indicate histopathological alterations, as described in the Results and Material and Methods sections. 
Turquoise module

\begin{tabular}{|c|c|c|c|c|c|c|}
\hline Cat. & Gene & GO database: biological process; molecular function & E-IPI & GCL3 & GCL $0-1$ & SEFI \\
\hline \multirow{4}{*}{ HHub } & PIK3C3 & protein kinase activity & -0.52 & -0.69 & 0.59 & -0.52 \\
\hline & DERA & deoxyribonucleotide catabolism & -0.57 & -0.70 & 0.52 & -0.50 \\
\hline & BUD13 & mRNA splicing, via spliceosome & -0.55 & -0.73 & 0.53 & -0.50 \\
\hline & $R B M \times 2$ & mRNA splicing, via spliceosome & -0.57 & -0.76 & 0.56 & -0.54 \\
\hline iHub & KIAA0020 & regulation of protein ADP-ribosylation & -0.56 & .0 .74 & 0.60 & -0.45 \\
\hline \multirow{4}{*}{ eHub } & SYF2 & mRNA splicing, via spliceosome & -0.58 & -0.72 & 0.49 & -0.56 \\
\hline & DCP1B & deadenylation-independent decapping of nuclear-transcribed mRNA & -0.59 & -0.74 & 0.53 & -0.61 \\
\hline & ZNF92 & regulation of transcription by RNA polymerase II & 0.57 & 0.63 & -0.60 & NS \\
\hline & ZNF91 & negative regulation of transcription, DNA-templated & 0.59 & 0.76 & -0.54 & 0.47 \\
\hline
\end{tabular}

\section{Brown module}

\begin{tabular}{|l|l|l|c|c|}
\hline Cat. & Gene & \multicolumn{1}{|c|}{ GO database: biological process; molecular function } & GCL3 & SMI \\
\hline \multirow{5}{*}{ iHub } & VPS26A & retromer complex & -0.59 & -0.45 \\
& CUL2A & protein ubiquitination & -0.67 & NS \\
& TOMM70A & protein targeting to mitochondrion & -0.65 & -0.49 \\
& NR2C1 & negative regulation of transcription by RNA polymerase II & -0.73 & -0.66 \\
& ARHGAP18 & regulation of small GTPase mediated signal transduction & -0.60 & -0.55 \\
\hline \multirow{4}{*}{ eHub } & VRK1 & protein kinase activity & -0.69 & -0.69 \\
& EIF4E & regulation of translation & -0.73 & -0.50 \\
& LGR4 & positive regulation of canonical Wnt signaling pathway & -0.83 & NS \\
& LYNC2LI1 & intraciliary retrograde transport & -0.70 & NS \\
\hline
\end{tabular}

\section{Black module}

\begin{tabular}{|l|l|l|c|}
\hline Cat. & Gene & \multicolumn{1}{|c|}{ GO database: biological process; molecular function } & GCL3 \\
\hline \multirow{2}{*}{ HHub } & SPCS2 & signal peptidase & -0.76 \\
& PAN3 & RNA phosphodiester bond hydrolysis, exonucleolytic & -0.76 \\
& NOC3L & chromatin binding & -0.75 \\
\hline \multirow{2}{*}{ iHub } & MGAT2 & protein N-linked glycosylation via asparagine & -0.79 \\
& MORF4L1 & histone deacetylation & -0.74 \\
\hline eHub & RUNDC1 & activation of GTPase activity & -0.64 \\
\hline
\end{tabular}

\section{Purple module}

\begin{tabular}{|c|l|l|c|}
\hline Cat. & Gene & \multicolumn{1}{|c|}{ GO database: biological process; molecular function } & GCD2 \\
\hline \multirow{2}{*}{ iHub } & HCRT & neuropeptide hormone activity & -0.55 \\
& SDK2 & $\begin{array}{l}\text { synapse assembly } \\
\text { proteolysis }\end{array}$ & -0.58 \\
& CTRB2 & -0.64 \\
\hline \multirow{2}{*}{ eHub } & FGD1 & regulation of GTPase activity & -0.49 \\
& MUC2 & $\begin{array}{l}\text { protein binding } \\
\text { histone deacetylation }\end{array}$ & -0.63 \\
& MIER2 & & -0.58 \\
\hline
\end{tabular}

Red module

\begin{tabular}{|l|l|l|c|}
\hline Cat. & Gene & \multicolumn{1}{|c|}{ GO database: biological process; molecular function } & E-Onset \\
\hline HHub & OLFM1 & regulation of axon extension & 0.60 \\
\hline \multirow{2}{*}{ iHub } & ST8SIA3 & N-glycan processing & 0.53 \\
& SVOP & synaptic vesicle; transmembrane transport & 0.55 \\
\hline \multirow{2}{*}{ eHub } & NCDN & neuron projection development & 0.47 \\
& ERBB3 & $\begin{array}{l}\text { neuregulin binding } \\
\text { brain development }\end{array}$ & -0.53 \\
\hline
\end{tabular}

\section{Figure 5}

High hierarchy genes (Hhubs, iHubs, and eHubs) of the meta-module II present in modules highly associated with at least one trait. Each $\mathrm{HH}$ gene is identified by its hierarchical categorization and $\mathrm{GO}$ biological process or molecular function. Genes that are HH and HGS (see Table 2) appear in red lettering. Columns in red or in green indicate genes with positive (i.e. hyper-expressed) or negative (i.e. hypoexpressed) GS values for a specific trait, respectively. NS indicates gene with non-significant $(p \geq 0.05)$ GS value for the specific trait. E-IPI stands for early IPI; E-Onset stands for early onset; SEFI stands for 
severe executive function impairment; SMI stands for severe memory impairment. The abbreviations GCD2, GCL0-1, GCL3 - refer to histopathological alterations, as described in the Results and Material and Methods sections.

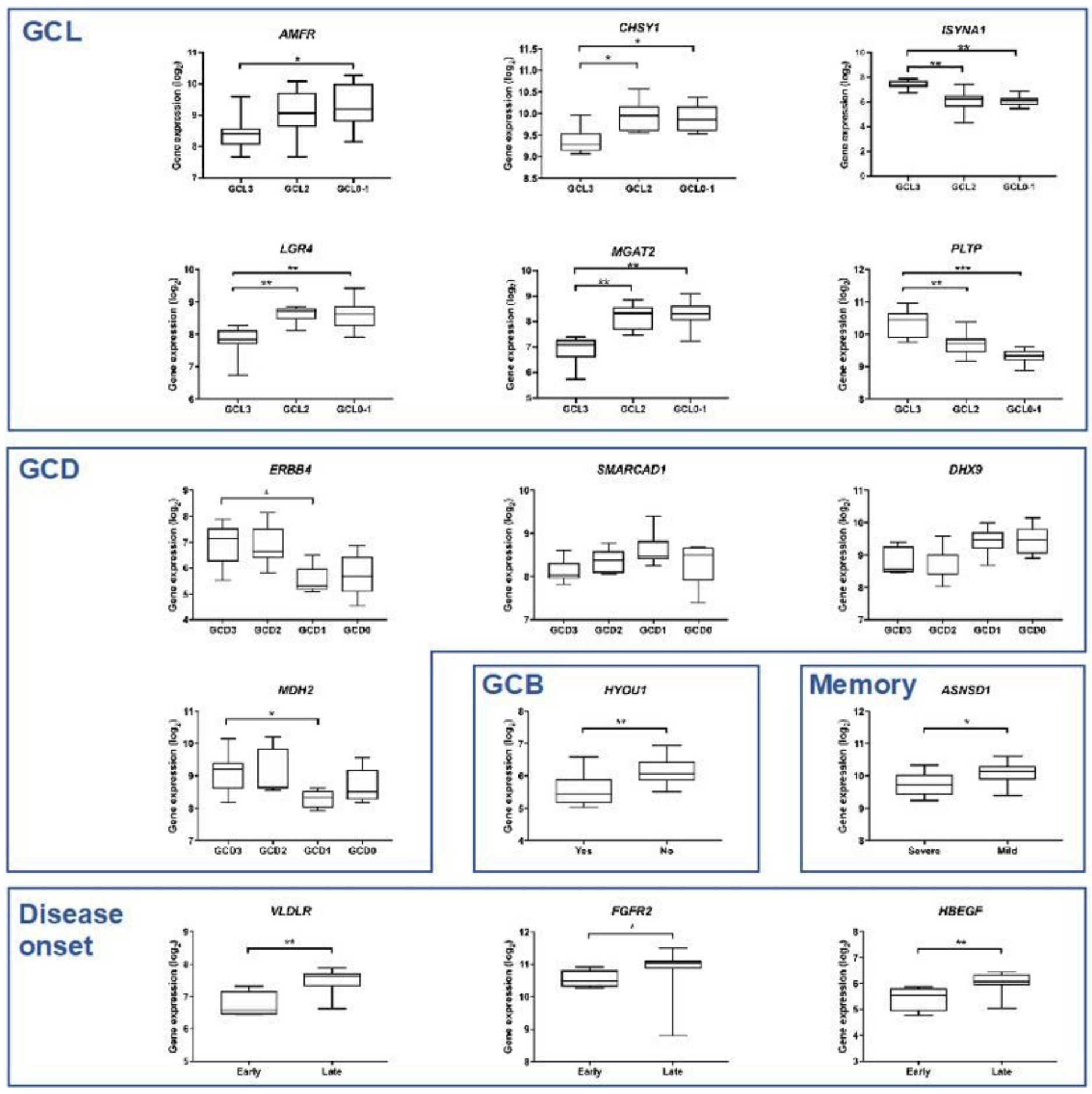

\section{Figure 6}

Gene expression plots for the HGS genes highly associated with specific histopathological or clinical data. The abbreviations - GCL, GCD, GCB - refer to histopathological alterations, as described in the Results and Material and Methods sections. Statistical significance values: ${ }^{\star} p<0.05 ;{ }^{* \star} p<0.005$. 


\section{Supplementary Files}

This is a list of supplementary files associated with this preprint. Click to download.

- BandoetalSupplementaryDatasheetS1andTablesS16.xIsx

- BandoetalSupplementaryFiguresS15.pdf 